\title{
Shape-Based Myocardial Contractility Analysis Using Multivariate Outlier Detection
}

\author{
Karim Lekadir ${ }^{1}$, Niall Keenan ${ }^{2}$, Dudley Pennell ${ }^{2}$, and Guang-Zhong Yang ${ }^{1}$ \\ ${ }^{1}$ Visual Information Processing, Department of Computing, Imperial College London, UK \\ ${ }^{2}$ Cardiovascular Magnetic Resonance Unit, Royal Brompton Hospital, London, UK
}

\begin{abstract}
This paper presents a new approach to regional myocardial contractility analysis based on inter-landmark motion (ILM) vectors and multivariate outlier detection. The proposed spatio-temporal representation is used to describe the coupled changes occurring at pairs of regions of the left ventricle, thus enabling the detection of geometrical and dynamic inconsistencies. Multivariate tolerance regions are derived from training samples to describe the variability within the normal population using the ILM vectors. For new left ventricular datasets, outlier detection enables the localization of extreme ILM observations and the corresponding myocardial abnormalities. The framework is validated on a relatively large sample of 50 subjects and the results show promise in localization and visualization of regional left ventricular dysfunctions.
\end{abstract}

\section{Introduction}

Assessment of the left ventricle is important to the management of patients with cardiac disease. With increasing advances in imaging techniques, most modalities now offer routine $4 \mathrm{D}$ coverage of the heart, allowing both global and local assessment of left ventricular morphology and function. Several existing semi- and fully-automatic segmentation methods allow rapid and objective delineation of the left ventricle [1-3]. Extracting relevant and reliable indices of myocardial contractile abnormality, however, remains a complex task [4-7]. Global markers such as stroke volume and ejection fraction are widely used in clinical practice but they are not suitable for identifying local abnormalities. Alternative regional assessment based on wall thickening is problematic for a number of reasons. First, important information such as shape, size and endo-cardial displacement are not encoded for dysfunction analysis. Additionally, only end-diastole and end-systole differences are taken into account, whilst certain symptoms such as cardiac dys-synchronization are related to the entire cardiac cycle. The definition of normal ranges is a further challenge as significant overlap exists with abnormal values. Furthermore, local assessment methods do not consider the geometry and motion at other locations of the left ventricle to detect inconsistencies. For these reasons, visual assessment by expert observers remains the gold standard in routine clinical applications, but it is time consuming and can involve significant bias.

The proposed method for myocardial abnormality localization is based on interlandmark motion (ILM) vectors, which represent the simultaneous endo- and epicardial changes occurring at two regions of the left ventricle over the entire cardiac 
cycle. By combining pairs of locations of the left ventricle in the spatio-temporal representation, geometrical and dynamic inconsistencies can be identified efficiently, whilst the overlap between normal and abnormal values is reduced significantly. Additionally, ILM vectors can implicitly incorporate shape, size, thickness, and endo-cardial displacement for dysfunction analysis. To describe the variability within the normal population, multivariate tolerance regions are derived from training samples in a robust manner. For a given left ventricular dataset, an abnormality likelihood measure is estimated for each location from its associated ILM vectors and an iterative procedure enables the localization of myocardial abnormality. The method is validated with a population of 50 datasets containing normal and abnormal cases.

\section{Methods}

\subsection{Inter-Landmark Motion (ILM) Vectors}

Conventional local assessment methods for abnormality localization consider each region of the left ventricle independently. Therefore, they do not take into account the global geometry and dynamics of the left ventricle in the analysis, nor in the definition of the normal ranges, thus causing significant overlap with abnormal values. To overcome these difficulties, this paper introduces inter-landmark motion (ILM) vectors, which describe the coupled motion and geometry over the entire cardiac cycle of pairs of myocardial locations (represented by landmark points). With this approach, each region is analyzed with respect to other locations of the left ventricle, allowing their coupled spatio-temporal relationships to be used for identifying geometrical or dynamic inconsistencies. Although the pairs of locations can be chosen for the entire left ventricle, it is more appropriate to restrict this to be within the same cross-section, where there is a high covariance between the landmarks. For each of the $m$ points within the same cross-section, $m-1$ ILM vectors can be derived.

In the proposed framework, the required landmark-based representation of the myocardial boundaries is first obtained through delineation or segmentation. For each myocardial location (landmark), two rotational and translational invariant descriptors are extracted, i.e., the distances of the endo- and epi-cardial borders to a reference point on the same cross-section plane. The reference point is chosen as the center of the epi-cardial border as it is less susceptible to morphological variations. The invariance to scaling is not considered to allow the detection of size related abnormalities, such as dilatation. Each ILM vector, of dimension $p=4 F$, can be written as follows:

$$
v\left(P_{i}, P_{j}\right)=\left(a_{i 1}, b_{i 1}, \ldots, a_{i F}, b_{i F}, \ldots, a_{j 1}, b_{j 1}, \ldots, a_{j F}, b_{j F}\right)^{T}
$$

where $F$ is the number of frames in the cardiac cycle and $a$ and $b$ denote the endoand epi-cardial variables, respectively. The ILM vectors provide, in addition to size and thickness measures encapsulated by these variables, an implicit description of the shape of the myocardial borders. 


\subsection{Multivariate Tolerance Regions}

In this work, the normal myocardial contractility properties are described using multivariate tolerance regions for each ILM vector. Given $N$ training samples, a tolerance region $T R$ in the $p$ dimensional space can be described as:

$$
T R=\left\{v \in \mathbb{R}^{p} \mid d(v, \mu, \Sigma)<L\right\}
$$

where $\mu$ and $\Sigma$ represent the location and scale of the multivariate distribution, $d$ a distance measure to the center of the distribution and $L$ a threshold that limits the size of the tolerance region to normal observations. The variability within the normal population can be well approximated by a multivariate normal distribution, in which case the location and scale in Eq. (1) are replaced by the mean observation $\bar{v}$ and the covariance matrix $S_{v}$, respectively. It was shown that an appropriate distance measure for multivariate normal tolerance regions is the Mahalanobis distance [8], i.e.,

$$
d\left(v, \bar{v}, S_{v}\right)=(v-\bar{v})^{T} S_{v}^{-1}(v-\bar{v})
$$

In order to consider only the principle modes of variation, an eigen-decomposition of the covariance matrix can be applied. By rejecting the $p-t$ noisy directions, the distance measure can be simplified to:

$$
d\left(v, \bar{v}, S_{v}\right)=\sum_{i=1}^{t} \frac{\left[U_{i}(v-\bar{v})_{i}\right]^{2}}{E_{i}} \text { where } S_{v}=U E U^{T}
$$

For the tolerance region limit $L$ in Eq. (2), it was shown that it can be estimated from the critical values of the chi-square distribution as [10]:

$$
L=\chi_{t,(1-\alpha)^{1 / N}}^{2}
$$

A training sample of normal subjects is used to capture the normal variability of myocardial contractility. In practice, however, extreme values of the ILM vectors may arise from some unexpected local abnormalities or due to errors in boundary delineation. This can considerably affect the calculation of the mean and covariance matrix. Therefore, a robust estimation of the tolerance region parameters is required.

A natural robust estimator for the central observation of the distribution can be found by replacing the mean by the median vector, denoted as $v^{*}$. A weighted estimation of the covariance can then be achieved in an iterative manner, where the robust covariance matrix $S_{v}^{*}$ at iteration $t+1$ is calculated as:

$$
S_{v}^{*}(t+1)=\frac{\sum_{i=1}^{N} w^{2}\left(v_{i}, v^{*}, S_{v}^{*}(t)\right)\left(v_{i}-v^{*}\right)\left(v_{i}-v^{*}\right)^{T}}{\sum_{i=1}^{N} w\left(v_{i}, v^{*}, S_{v}^{*}(t)\right)}
$$


where $w$ is a weight calculated from the observation, the median and the covariance matrix at previous iteration. The idea behind this formulation is to weight equally and heavily the observations that are close to the median and decrease the weights for observation further away. This procedure is repeated until the values of the weights do not change significantly. A definition of the weights can be written as follows:

$$
w\left(v, v^{*}, S^{*}\right)=\left\{\begin{array}{cc}
1 & \text { if } d\left(v, v^{*}, S^{*}\right)<d_{0} \\
\exp \left(-\frac{\left(d\left(v, v^{*}, S^{*}\right)-d_{0}\right)^{2}}{2 \sigma_{0}^{2}}\right) & \text { elsewhere }
\end{array}\right.
$$

where $d_{0}$ is a threshold calculated from the median $d^{*}$ and the robust standard deviation $\sigma^{*}$ of all distances [9], and $\sigma_{0}$ specifies the decay rate for distances above the threshold, i.e.,:

$$
\begin{aligned}
& d_{0}=d^{*}+c_{1} \sigma^{*}\left(2 \leq c_{1} \leq 3\right) \text { and } \sigma_{0}=c_{2} \sigma^{*}\left(0<c_{2}<1\right) \\
& d^{*}=\underset{1 \leq i \leq N}{\operatorname{median}}\left(d\left(v, v^{*}, S^{*}\right)\right) \text { and } \sigma^{*}=1.4826 \underset{1 \leq i \leq N}{\operatorname{median}}\left|d\left(v, v^{*}, S^{*}\right)-d^{*}\right|
\end{aligned}
$$

\subsection{Contractile Abnormality Identification}

For a given left ventricular dataset with delineated boundaries, the ILM vectors in Eq. (1) are calculated and outlying vectors are identified by using the following measure:

$$
f_{v}(v)= \begin{cases}1 & \text { if } v \in T R \\ 0 & \text { if } v \notin T R\end{cases}
$$

Because each ILM vector incorporates a pair of myocardial locations, it is not straightforward to identify which of the two landmarks corresponds to an abnormality when the vector in question is outside of the tolerance region. Abnormal landmarks, however, will have a high level of invalid ILM vectors. Therefore, a likelihood measure of abnormality can be calculated for each landmark by summing all measures from Eq. (9) for all the associated ILM vectors, i.e.,

$$
f_{p}(P)=1-\frac{1}{m-1} \sum_{i=1}^{m-1} f_{v}\left(v_{i}\right)
$$

An iterative procedure is then used, with which the landmark with the highest abnormality measure is identified as abnormal. The abnormality measures of the remaining landmarks are updated by subtracting the contribution of the rejected landmark. The procedure is repeated until the highest abnormality measure is close to 0 , suggesting that all remaining landmarks correspond to normal myocardial contractility. 


\subsection{Validation}

The validation of the technique is carried out on a relatively large sample of 50 leftventricular datasets. The subjects were scanned using a 1.5T MR scanner (Sonata, Siemens, Erlangen Germany) and a TrueFISP sequence (TE $=1.5 \mathrm{~ms}$, TR $=3 \mathrm{~ms}$, slice thickness $=10 \mathrm{~mm}$ and pixel size from 1.5 to $2 \mathrm{~mm}$ ) within a single breath-hold. Retrospective cardiac gating was used to ensure an even coverage of the entire cardiac cycle and for each subject 25 cine frames were acquired.

For all datasets, delineation of the myocardial boundaries was carried out by an expert clinician by using a semi-automatic ventricular analysis tool. From the obtained contours, 182 landmarks were uniformly distributed by arc length for each of the endo- and epi-cardial shapes, where point correspondences were determined based on the location of the LV/RV junction points. Ejection fraction, stroke volumes and thickening were calculated and a detailed visual assessment was carried out by the expert observer for dysfunction localization. The 50 subjects were classified as normal, mildly/intermediately abnormal or severely abnormal. In this study, a total of 28 subjects were identified as normal by the expert observer and used for the tolerance model construction. All datasets were then evaluated using the proposed method, where the normal subjects were assessed on a leave-one-out basis.

\section{Results}

The percentage of abnormal landmarks was calculated for each dataset and plotted in Fig. 1 against the visual classification ((a) normal, (b) mildly/intermediately abnormal and (c) severely abnormal). It can be seen from the figure that the calculated percentage of abnormality correlates well with the visual classification and that a good separation is achieved for almost all datasets. For numerical assessment of class separation, non-parametric tests were used and a significant difference between the 3 groups was found using the Kruskal-Wallis test $(\mathrm{p}<0.001)$ and post-hoc multiple



Fig. 1. Percentage of abnormality as calculated by the proposed technique for the entire datasets, plotted against the visual classification by the expert observer 
comparisons using Mann-Whitney test showed significant differences between each of the 3 groups $(\mathrm{p}<0.001)$. The average abnormality percentage found for the normal, mildly abnormal and severely abnormal subjects were $1.0 \pm 2.0,14.2 \pm 6.0$ and $63.8 \pm$ 25.1 , respectively. Two normal datasets (shown in crosses) were misclassified by the proposed technique. The first one, characterized by extreme thickening of the myocardium (probably due to stress during the scan), was misclassified because of a training sample that did not include the corresponding variability. The second misclassification is due to right ventricular dysfunction and is discussed in detail in Fig. 4(b).

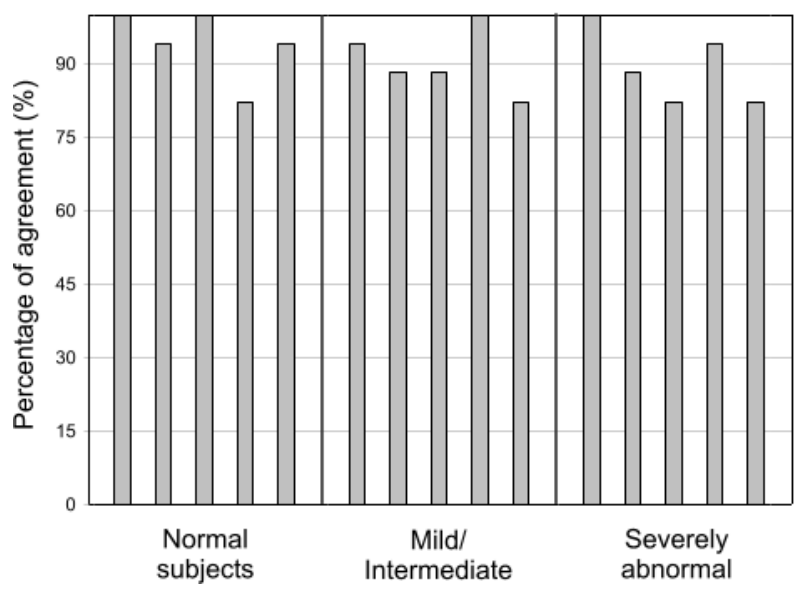

Fig. 2. Percentage agreement between automatic and manual dysfunction analysis for the normal and abnormal segments

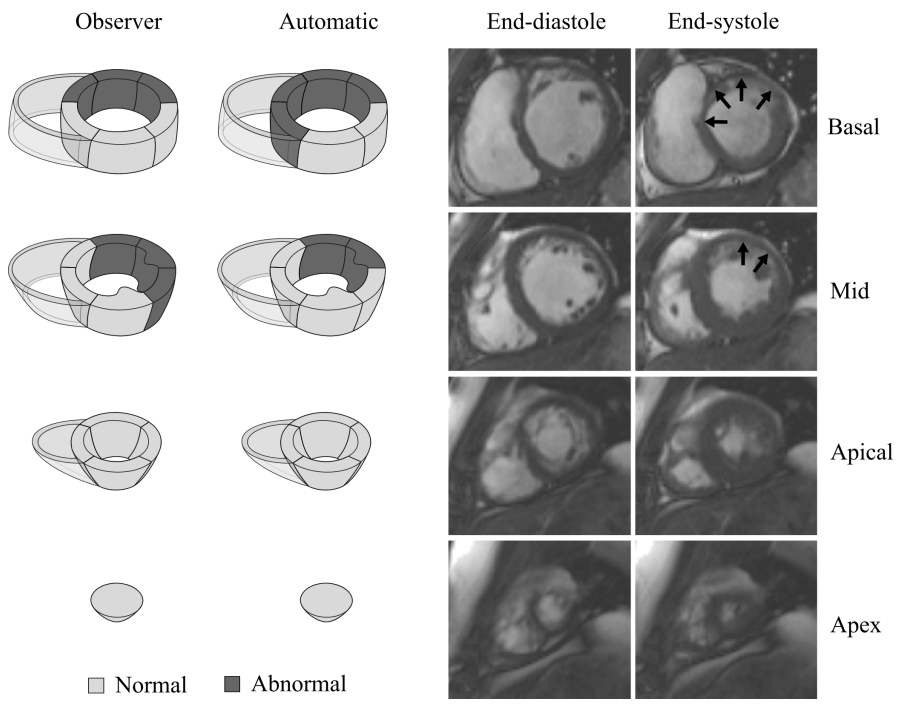

Fig. 3. An example comparing the 17-segment based local assessment achieved by the automatic and manual abnormality analysis methods 
(a)

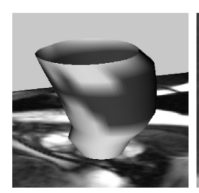

LA-ED

LA-ES

SA-ED SA-ES

(b)
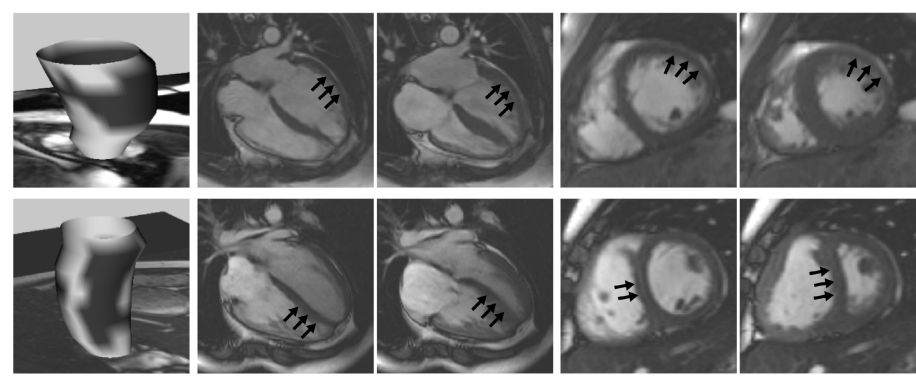

(c)
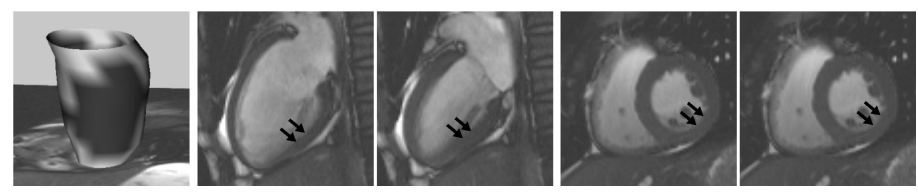

Fig. 4. Three examples illustrating the dysfunction analysis achieved by the proposed method. The results are mapped onto the LV surface for abnormality localization and visualization.

For local assessment of the technique, five datasets were selected from each of the three classes and further analyzed using the American Heart Association/American College of Cardiology (AHA/ACC) recommended 17-segment model. The segments were classified by the expert as normal or abnormal and for the proposed method, the abnormality measures were averaged for each segment. By counting the number of misclassifications, a percentage of agreement between the proposed method and the visual classification was calculated for the fifteen datasets and plotted in Fig. 2. It is evident from the graph that a good agreement was achieved throughout the datasets, with an average percentage equal to $91.3 \pm 6.9 \%$. Fig. 3 shows an example of the segmental assessment achieved by both the automatic and manual methods. The identified abnormal segments correspond to myocardial infarct as it can be seen on the short axis images. The manual and automatic classifications correspond well overall.

To visualize the localization of the abnormalities, LV surface maps were constructed using the results of the proposed abnormality detection technique. Three examples are displayed in Fig. 4, where the lighter shading corresponds to normal myocardial contractility while the darker shading indicates a local abnormality. The example in (a) is a left ventricle with partial dilatation and abnormal ejection fraction (36\%). Due to a formation of scar tissue at the antero-lateral region, the wall does not thicken as shown on the MR images and as identified by the abnormality map. The example in (b) corresponds to the second misclassification from Fig. 1, with normal ejection fraction and thickening measures. The subject, however, has an abnormal right ventricle which is affected by pulmonary hypertension, and thus pushes into the left ventricle. This causes a severe deformation at the septal region of the LV, which is correctly identified by the proposed method. In (c), a normal thickening is found overall, but it is less significant at the infero-lateral region than at the other regions of the myocardium. This is usually suspicious of myocardial ischemia. 


\section{Conclusion}

This paper presents a new model-based approach to myocardial contractility analysis, capable of detecting shape and motion inconsistencies between myocardial regions by using inter-landmark motion vectors and multivariate outlier detection. The results obtained on a relatively large sample show promise in localization and visualization of regional left ventricular dysfunctions. Future work includes the validation of the method on different groups of patients to enhance its clinical value, as well as its application to right ventricular dysfunction analysis.

\section{References}

1. Mitchell, S.C., Bosch, J.G., Lelieveldt, B.P.F., Geest, R.J.V.D., Reiber, J.H.C., Sonka, M.: 3-D active appearance models: segmentation of cardiac MR and ultrasound images. IEEE Transactions on Medical Imaging 21, 1167-1178 (2002)

2. Lekadir, K., Merrifield, R., Yang, G.-Z.: Outlier detection and handling for robust 3D active shape models search. IEEE Transactions on Medical Imaging 26, 212-222 (2007)

3. Jolly, M.-P., Duta, N., Funka-Lea, G.: Segmentation of the left ventricle in cardiac MR images. In: International Conference on Computer Vision (ICCV) (2001)

4. Frangi, A.F., Niessen, W.J., Viergever, M.A.: Three-dimensional modeling for functional analysis of cardiac images: a review. IEEE Transactions on Medical Imaging 20, 2-25 (2001)

5. Suinesiaputra, A., Üzümcü, M., Frangi, A.F., Kaandorp, T.A.M., Reiber, J.H.C., Lelieveldt, B.P.F.: Detecting regional abnormal cardiac contraction in short-axis MR images using independent component analysis. In: Barillot, C., Haynor, D.R., Hellier, P. (eds.) MICCAI 2004. LNCS, vol. 3216, Springer, Heidelberg (2004)

6. Declerck, J., Feldmar, J., Ayache, N.: Definition of a 4D continuous planispheric transformation for the tracking and the analysis of LV motion. Medical Image Analysis 2, 197$213(1998)$

7. Shi, P., Sinusas, A.J., Constable, R.T., Ritman, E., Duncan, J.S.: Point-tracked quantitative analysis of left ventricular surface motion from 3D image sequences: Algorithms and validation. IEEE Transactions on Medical Imaging 19, 36-50 (2000)

8. Healy, M.J.R.: Multivariate normal plotting. Applied Statistics 17, 157-161 (1968)

9. Huber, P.J.: Robust statistics. Wiley, New York (1981)

10. Becker, C., Gather, U.: The masking breakdown point of multivariate outlier identification rules. Journal of the American Statistical Association 94, 947-955 (1999) 